\title{
On the Spin content of the Nucleon
}

\author{
N. F. Nasrallah* \\ Faculty of Sciense, Lebanese University \\ Tripoli, Lebanon.
}

\begin{abstract}
A QCD sum rule calculation of Balistky and Ji on the spin content of the nucleon is done with a different approach to the evaluation of the bilocal contributions and to the extraction of the nucleon pole residues. The result obtained is much more numerically stable which puts their conclusion that more than half of the nucleon spin is carried by gluons on firmer ground.
\end{abstract}

The question of the spin distribution of the nucleon among its constituents has recently received much attention and it is now realized that a substantial fraction of the nucleon spin is carried by the quark orbital and gluon angular momenta [1]

$$
\frac{1}{2}=\frac{1}{2} \Delta \Sigma\left(\mu^{2}\right)+L_{q}\left(\mu^{2}\right)+J_{q}\left(\mu^{2}\right)
$$

Where $\mu^{2}$ is the scale at which the relevant operators are renormalized (we shall take here $\mu^{2} \simeq 1 \mathrm{GeV}^{2}$ and omit it from the notation). The first term in eq.(1) has been measured in polarized deep inelastic scattering [2]. The second and third terms represent quark orbital and gluon contributions respectively.

Ji [3] has recently shown that the total quark (and hence gluon) contribution to the nucleon spin is measurable through virtual Compton scattering in a special kinematic region where single quark scattering dominates via the study of off forward parton distributions (OFPD) whose second moment yields the quark contribution.

It was further shown by Vanderhaegen, Guichon and Guidal [4] that hard electroproduction of photons and mesons on the nucleon also gives access to the OFPD.

These authors also suggest that preliminary measurements can soon start at HERMES and CEBAF which can shed light on the spin content of the proton.

*e-mail: nsrallh@cyberia.net.lb 
An explicitly gauge invariant decomposition of the angular momentum operator in QCD was first given by Ji [3]

$$
\vec{J}_{\mathrm{QCD}}=\int\left[\frac{1}{2} \vec{\psi} \vec{\gamma} \gamma_{5} \psi+\psi^{+} \vec{x} \times i \vec{D} \psi+\vec{x} \times \vec{E} \times \vec{B}\right] d^{3} x
$$

This has subsequently been used by Balitsky and Ji [5] to study the three point function

$$
W_{g}^{\mu \nu \alpha}(p) \underset{\lim q \rightarrow 0}{=} \int \exp (i p x) \exp (i q z)\left\langle 0\left|T \eta(x) \bar{\eta}(0) M_{g}^{\mu \nu \alpha}(z)\right| 0\right\rangle d x d z
$$

where $\eta$ is the nucleon field [6]

$$
\begin{gathered}
\eta(x)=\epsilon^{i j k}\left(u^{i T} c \gamma^{\alpha} u^{j}\right) \gamma_{5} \gamma_{\alpha} d^{k} \\
M^{\mu \gamma \alpha}=T^{\mu \alpha} x^{\nu}-T^{\mu \nu} x^{\alpha} \text { is the angular momentum density } \\
T^{\alpha \beta}=T_{q}^{\alpha \beta}+T_{g}^{\alpha \beta}=\frac{1}{4} \bar{\psi} \gamma^{(\alpha} i \overleftrightarrow{D}^{\beta)} \psi+\frac{1}{4}\left(g^{\alpha \beta} F^{2}-F^{\alpha \mu} F^{\beta} \mu\right)
\end{gathered}
$$
with

The energy momentum tensor of QCD written as the sum of its quark and gluon parts $((\alpha \beta)$ means symmetrization in the indices)

$$
W_{g}^{\mu \nu \alpha}=W\left(p^{2}\right)\left(2 i p^{\mu} \gamma^{\nu} \nu^{\alpha}\right)+\text { other tensor structures }
$$

$W\left(p^{2}\right)$ contain nucleon double and single pole contribution as well as a non singular contribution of the continuum

$$
W\left(p^{2}\right)=\frac{J_{g} \lambda_{N}^{2}}{\left(p^{2}-m_{N}^{2}\right)^{2}}+\frac{c^{\prime}}{\left(p^{2}-m_{N}^{2}\right)}+\cdots
$$

$\lambda_{N}$ is the coupling of the nucleon to the current $\eta$

$$
\langle 0|\eta| N(p)\rangle=\lambda_{N} u(p)
$$

Using a Ward identity the 3-point function(3) is rewritten as

$$
\begin{gathered}
W_{g}^{\mu \nu \alpha} \underset{\lim q \rightarrow 0}{=} \int d x d z z^{\mu} z^{\nu} \exp (i p x) \exp (i q y)\langle 0| \\
O^{\alpha}(z)=\bar{\psi}_{f} F^{\alpha \beta} \gamma_{\beta} \psi(z)
\end{gathered}
$$


In the deep Euclidean region $W$ can be calculated in QCD

$$
\begin{aligned}
W^{\mathrm{QCD}}= & \frac{\alpha_{s}}{\pi^{s}}\left[\frac{1}{144} \ln ^{2}\left(-\frac{p^{2}}{\mu^{2}}\right)-\frac{1}{36} \ln \left(-\frac{p^{2}}{\mu^{2}}\right)\right] p^{2} \\
& -\frac{1}{144 \pi^{2} p^{2}}\left\langle\frac{\alpha_{s} F^{2}}{\pi}\right\rangle\left[\ln \left(-\frac{p^{2}}{\mu^{2}}\right)+\frac{7}{6}-\ln \left(-\frac{q^{2}}{\mu^{2}}\right)\right] \\
& -\frac{1}{81 \pi p^{4}} \alpha_{s}\langle\bar{q} q \bar{q} q\rangle\left[20 \ln \left(-\frac{p^{2}}{\mu^{2}}\right)-62 \ln \left(-\frac{q^{2}}{\mu^{2}}\right)\right]+\cdots
\end{aligned}
$$

The terms in $\ln \left(-\frac{q^{2}}{\mu^{2}}\right)$ signal the breakdown of the validity of the OPE for $q^{2} \longrightarrow 0$, they have to be replaced by bilocal contributions [5], i.e. one first expands $T \eta(x) \eta(0)=\sum c_{n}(x) O_{n}$ for small values of $\mathrm{x}$, the operators $O_{n}$ then combine with $O^{\alpha}(z)$ in a series of two point functions the leading terms in the asymptotic behaviour of which reproduce the terms in $\ln \left(-\frac{q^{2}}{\mu^{2}}\right)$ and which must be extrapolated to $q^{2}=0$. Expression(8) must then be replaced by

$$
\begin{aligned}
W^{\mathrm{QCD}}= & \frac{\alpha_{s}}{\pi^{5}}\left[\frac{1}{144} \ln ^{2}\left(-\frac{p^{2}}{\mu^{2}}\right)-\frac{1}{36} \ln \left(-\frac{p^{2}}{\mu^{2}}\right)\right] p^{2} \\
& -\frac{1}{144 \pi^{2} p^{2}}\left\langle\frac{\alpha_{s} F^{2}}{\pi}\right\rangle\left[\ln \left(-\frac{p^{2}}{\mu^{2}}\right)+\frac{7}{6}\right]-\frac{1}{12 \pi^{2} p^{2}} \Pi_{0}\left(q^{2}=0\right) \\
& +\frac{20}{81 \pi p^{4}} \alpha_{s}\left\langle\bar{q} q^{2}\right\rangle \ln \left(-\frac{p^{2}}{\mu^{2}}\right)+\frac{4}{3 p^{4}} \Pi_{1}\left(q^{2}=0\right)
\end{aligned}
$$

where

$$
\Pi_{0,1}\left(q^{2}\right)=\int d z \exp (i q z)\left\langle 0\left|T O_{5,7}(0) O^{\alpha}(z)\right| 0\right\rangle
$$

and the relevant operators

$$
\begin{aligned}
O_{5}^{\lambda \rho \rho^{\prime}}= & 2 \bar{u} g F^{\lambda[\rho} \gamma^{\left.\rho^{\prime}\right]} u-2 i \partial^{[\rho}\left(\bar{u}^{\overleftrightarrow{D}}{ }^{\lambda} \gamma^{\left.\rho^{\prime}\right]} u\right) \\
& +\bar{u} \overleftarrow{\gamma^{\mu} D_{\mu}} \vec{D}^{\lambda} \sigma^{\rho \rho^{\prime}} \overleftarrow{D^{\lambda}} \overrightarrow{\gamma^{\mu} D_{\mu}} u \\
& +\frac{3}{4} \bar{u} g F^{\rho \rho^{\prime}} \gamma^{\lambda} u+\frac{3}{4} \bar{d} g F^{\rho \rho^{\prime}} \gamma^{\lambda} d \\
O_{7}^{\lambda \rho \rho^{\prime}}= & \epsilon^{i j k} \epsilon^{i^{\prime} j^{\prime} k^{\prime}}\left(D^{\lambda} u\right)^{i} C \gamma^{\rho} u^{j} \bar{u}^{j^{\prime}} \gamma^{\rho^{\prime}} C \bar{u}^{i^{\prime} T}+\text { h.c. }
\end{aligned}
$$

In the deep Euclidean region

$$
\Pi_{0}^{\mathrm{QCD}}\left(s=q^{2}\right)=-\frac{\alpha_{s}}{60 \pi^{3}} s \ln (-s)-\frac{1}{12}\left\langle\frac{\alpha_{s} F^{2}}{\pi}\right\rangle \ln (-s)+\frac{8}{9} \pi \alpha_{s}\left\langle(\bar{q} q)^{2}\right\rangle \frac{1}{s}+\cdots
$$


The method followed here to extract $\Pi_{0}\left(q^{2}=0\right)$ differs from the one used in ref[5]. $\Pi_{0}(s)$ is an analytic function in the complex $s$-plane except for cut along the positive real axis. Consider the integral over the closed contour in the complex $s$-plane consisting of a large circle of radius $R_{1}$ and two straight lines immediately above and below the cut which run from threshold to $R_{1}$. By virtue of Cauchy's theorem

$$
\begin{aligned}
\frac{1}{2 \pi i} \int_{c} \frac{d s}{s}\left(m^{\prime 2}-s\right) \Pi_{0}(s) & =\frac{1}{\pi} \int_{t h}^{R} \frac{d s}{s}\left(m^{\prime 2}-s\right) \operatorname{Im} \Pi_{0}(s)+\frac{1}{2 \pi i} \oint \frac{d s}{s}\left(m^{\prime 2}-s\right) \Pi_{0}(s) \\
& =m^{\prime 2} \Pi_{0}(0)
\end{aligned}
$$

Where $m^{\prime}$ is a mass parameter. The integral over the real axis corresponds to hadronic intermediate states with quantum numbers $J^{p c}=1^{-+}$, as $m^{\prime 2}$ is varied from threshold to $R$ this integral changes sign which implies that it vanishes for some intermediate value of $m^{\prime 2}$ which is expected to be close to the value of $s$ for which $\operatorname{Im} \Pi_{0}(s)$ reaches its maximum. Experimentally the E857 collaboration [8] has recently observed a broad bump in the invariant mass squared of the $\eta \pi$ system produced in the reaction $\pi^{-} p-\longrightarrow \eta \pi^{-} p$ around $2.0 \mathrm{GeV}^{2}$. So if we take $m^{\prime 2} \simeq 2.0 \mathrm{GeV}^{2}$ we expect the integral over the real axis in eq.(13) to be negligible. In the integral over the circle $\Pi_{0}$ is well approximated by $\Pi_{0}^{\mathrm{QCD}}$ except possibly for a small region near the real axis. Eqs.(12) and (13) then yield

$$
\Pi_{0}\left(p^{2}=0\right) \simeq \frac{\alpha_{s} R_{1}^{2}}{60 \pi^{3}}\left(\frac{R_{1}}{3 m^{\prime 2}}-\frac{1}{2}\right)+\frac{1}{12}\left\langle\frac{\alpha_{s} F^{2}}{\pi}\right\rangle\left(\frac{R_{1}}{m^{\prime 2}}-\ln \frac{R_{1}}{\mu^{2}}\right)-\frac{8}{9} \pi \alpha_{s}\left\langle(\bar{q} q)^{2}\right\rangle \frac{1}{m^{\prime 2}}
$$

In a similar fashion the asymptotic behaviour of $\Pi_{1}(s)$

$$
\Pi_{1}^{\mathrm{QCD}}(s)=-\frac{31}{54} \frac{\alpha_{s}}{\pi}\left\langle\bar{q} q^{2}\right\rangle \ln \left(-\frac{s}{\mu^{2}}\right)+\langle g \bar{q} F \sigma q q q\rangle\left(\frac{1}{3 s}\right)+\cdots
$$

yields

$$
\Pi_{1}\left(q^{2}=0\right)=\frac{31}{54} \frac{\alpha_{s}}{\pi}\left\langle\bar{q} q^{2}\right\rangle\left(\frac{R_{1}}{m^{\prime 2}}-\ln \frac{R_{1}}{\mu^{2}}\right)+\frac{1}{3} \frac{m_{0}^{2}}{m^{\prime 2}}\left\langle(\bar{q} q)^{2}\right\rangle
$$

where we have parameterized

$$
\langle g \bar{q} F \sigma q q q\rangle=-m_{0}^{2}\left\langle(\bar{q} q)^{2}\right\rangle
$$

In the equations above $R_{1} \simeq 4 \mathrm{GeV}^{2}$ and $m_{0}^{2} \simeq .65 \mathrm{GeV}^{2}$ are reasonable estimates (5) 
We are now able to extract the residue $J_{g}$. This is done by considering the Laplace type integral [9] $\frac{1}{2 \pi i} \int_{c} d t \exp \left(-\frac{t}{M^{2}}\right) W\left(t=p^{2}\right)$ in the complex t-plane over a closed contour consisting of a circle of radius $\mathrm{R}$ and two straight lines above and below the cut which run from threshold to $R . M^{2}$ is the usual 'Borel mass' parameter. The exponential provides convenient damping of the integral over the continuum which we expect to be small for an appropriate choice of $M^{2}$. If this is done we get a relation between the residues of $W$ and the integral of $W^{\mathrm{QCD}}$ on the circle of radius $R$

$$
\begin{aligned}
J_{g}+c^{\prime} M^{2}= & \frac{1}{\lambda_{N}^{2} \exp \left(-\frac{m_{N}^{2}}{M^{2}}\right)}\left[\frac{\alpha_{s} M^{6}}{36 \pi^{5}}\left(1-\frac{1}{2} \ln \frac{R}{\mu^{2}}\right) \int_{0}^{\frac{R}{M^{2}}} d x x \exp (-x)\right. \\
& +\left[\frac{1}{144 \pi^{2}}\left\langle\frac{\alpha_{s} F^{2}}{\pi}\right\rangle \frac{7}{6}+\frac{1}{12 \pi^{2}} \Pi_{0}(0)\right] M^{2} \\
& +\frac{1}{144 \pi^{2}}\left\langle\frac{\alpha_{s} F^{2}}{\pi}\right\rangle \frac{M^{2}}{2 \pi i} \oint d t \exp \left(-\frac{t}{M^{2}}\right) \frac{\ln (-t)}{t} \\
& \left.-\frac{20 M^{2}}{81 \pi} \alpha_{s}\left\langle\bar{q} q^{2}\right\rangle \frac{1}{2 \pi i} \oint \frac{d t}{t^{2}} \exp \left(-\frac{t}{M^{2}}\right) \ln (-t)+\frac{4}{3} \Pi_{1}(0)\right]
\end{aligned}
$$

$\lambda_{N}^{2}$ is obtained from a study of the two point function [6]

$$
\begin{aligned}
\int d x & \exp (i q x)\langle 0|T \eta(x) \bar{\eta}(0)| 0\rangle \\
(2 \pi)^{4} \lambda_{N}^{2} \exp \left(-\frac{m_{N}^{2}}{M^{2}}\right)= & \frac{1}{4} M^{6} \int_{0}^{\frac{R}{M^{2}}} d x x^{2} \exp (-x)-\frac{\pi^{2}}{2}\left\langle\frac{\alpha_{s} F^{2}}{\pi}\right\rangle M^{2} \int_{0}^{\frac{R}{M^{2}}} d x \exp (-x) \\
& +\frac{32}{3} \pi^{4}\left\langle(\bar{q} q)^{2}\right\rangle
\end{aligned}
$$

The choice of $M^{2}$ in the equation above and the coherence of the method is dictated by stability considerations, if there are values of $M^{2}$ small enough to provide adequate damping of the continuum and large enough to justify the neglect of the contributions of higher order condensates in the OPE, this should show up in the linear behaviour of the r.h.s. of eq.(18) in some intermediate range of $M^{2}$. The standard value [9] $\left\langle\frac{\alpha_{s} F^{2}}{\pi}\right\rangle=0.012 \mathrm{GeV}^{2}$ is used, for the value of the condensate $\left\langle(\bar{q} q)^{2}\right\rangle$ the choice $\langle\bar{q} q\rangle^{2}$ (vacuum saturation hypothesis) is usually made but as it seems too stringent an assumption [10] we take $\left\langle(\bar{q} q)^{2}\right\rangle=\beta\langle\bar{q} q\rangle^{2}$ the dependance on $\beta$ is weak because it appears both in the numerator and in the denominator of the r.h.s. of eq.(18). In fig.1 the r.h.s. of eq.(18) is plotted as a function of $M^{2}$ for $\beta=1$. It is seen that the dependance on $M^{2}$ is essentially linear in the interval $.7 \mathrm{GeV}^{2} \lesssim M^{2} \lesssim 1.2 \mathrm{GeV}^{2}$ with a small value of the slope $\left(c^{\prime} \simeq 0\right)$. 
The result is

$$
J_{g} \simeq 0.35 \text { for } \beta=1, J_{g} \simeq 0.30 \text { for } \beta=3
$$

Balitsky and Ji [5] had reached the same conclusion, they had however chosen to eliminate the contribution of the nucleon single pole by multiplying $W$ by $\left(t-m_{N}^{2}\right)$ before performing the Borel transformation and used vector meson dominance to estimate $\Pi_{0,1}(0)$. This has the unfortunate effect of destabilizing the calculation as it can be seen that the variation of their value for $J_{g}$ with $M^{2}$ is very rapid.

This can be seen explicitely from the expression obtained following the method of ref.[5]. Instead of eq.(18) one gets

$$
\begin{aligned}
J_{g}= & \frac{1}{\lambda_{N}^{2} \exp \left(-\frac{m_{N}^{2}}{M^{2}}\right)}\left[\frac{-\alpha_{s} M^{6}}{36 \pi^{5}}\left(1-\frac{1}{2} \ln \frac{R}{\mu^{2}}\right) \int_{0}^{\frac{R}{M^{2}}} d x x\left(x-\frac{m_{N}^{2}}{M^{2}}\right) \exp (-x)\right. \\
& +\left[\frac{1}{144 \pi^{2}}\left\langle\frac{\alpha_{s} F^{2}}{\pi}\right\rangle \cdot \frac{7}{6}+\frac{1}{12 \pi^{2}} \Pi_{0}(0)\right] m_{N}^{2} \\
& -\frac{1}{144 \pi^{2}}\left\langle\frac{\alpha_{s} F^{2}}{\pi}\right\rangle \cdot \frac{1}{2 \pi i} \oint d t \exp \left(-\frac{t}{M^{2}}\right) \frac{\ln (-t)}{t}\left(t-m_{N}^{2}\right) \\
& +\frac{20}{81 \pi} \alpha_{s}\left\langle(\bar{q} q)^{2}\right\rangle \cdot \frac{1}{2 \pi i} \oint d t \exp \left(-\frac{t}{M^{2}}\right) \frac{\ln (-t)}{t}\left(t-m_{N}^{2}\right) \\
& \left.+\frac{4}{3} \Pi_{1}(0)\left(1+\frac{m_{N}^{2}}{M^{2}}\right)\right]
\end{aligned}
$$

stability here requires the r.h.s. of eq.(21) to be constant in an intermediate range of $M^{2}$. As can be seen from fig.2 no such thing happens. This confirms what has been stated above: elimination of the simple pole contribution by multipication by $\left(t-m_{N}^{2}\right)$ before perfoming the Borel multiformation destabilizes the calculation. The main difference between the approach advocated here and the one of ref. [5], in addition to the use of eq.(13) instead of vector meson dominance to evaluate the bilocal contributions $\Pi_{0,1}(0)$,consists in not multiplying by $\left(t-m_{N}^{2}\right)$ in order to restore numerical stability.

Acknowledgments: I thank the Academie de Versailles for its generous support and the IPN, Orsay, where the essential part of this work was done, for their kind hospitality 


\section{References}

[1] For a review see H.Y. Chang, Int. J. Mod. Phys. A11 (1996) 5109

R.L. Jaffe Report no. MIT-CTP-2518(1996), hep-ph/9603422.

[2] J. Ashman et al. Nucl. Phys. B328 (1989) 1

B. Adeva et al. Phys. Lett. B302 (1993) 533

P. L. Anthony et al. Phys. Rev. Lett. 71 (1993) 959

K. Abe et al. Phys. Rev. Lett. 78 (1995) 25

[3] X. Ji, Phys. Rev. Lett. 78 (1997) 610

[4] M. Vanderhaeghen, P. A. M. Guichon and M. Guidal, Phys. Rev. Lett. 80 (1998) 5064

[5] I. Balitsky and X. Ji, Phys. Rev. Lett. 79 (1997) 1225

[6] B. Ioffe, Z. Phys. C18 (1983) 67

[7] I. Balitsky, Phys. Lett. 114B (1983) 53

[8] D. R. Thompson et al. Phys. Rev. Lett. 79 (1997) 1630

H. Aoyagi et al. Phys. Lett. B314 (1993) 246

G. Beladidze et al. Phys. Lett. B313 (1993) 276

C. Amber et al. Phys. Lett. B333 (1994) 277

[9] M. A. Shifman, A. I. Vainstein and V. I. Zakharov, Nucl. Phys. B147 (1979) 385

[10] M. Davier, L. Girlanda, A. Höcker and J. Stern, Phys. Rev. D58 (1998) 096014. 


\section{Figure Captions:}

fig.1: The r.h.s.of eq.(18) plotted against $M^{2}$. The linear variation of $J_{g}+c^{\prime} M^{2}$ is apparent in the range $.7 \mathrm{GeV}^{2} \lesssim M^{2} \lesssim 1.2 \mathrm{GeV}^{2}$

fig.2: The r.h.s. of eq.(21) plotted against $M^{2} . J_{g}$ which ought to be constant in some range of $M^{2}$ varies rapidly. 
fig. 1

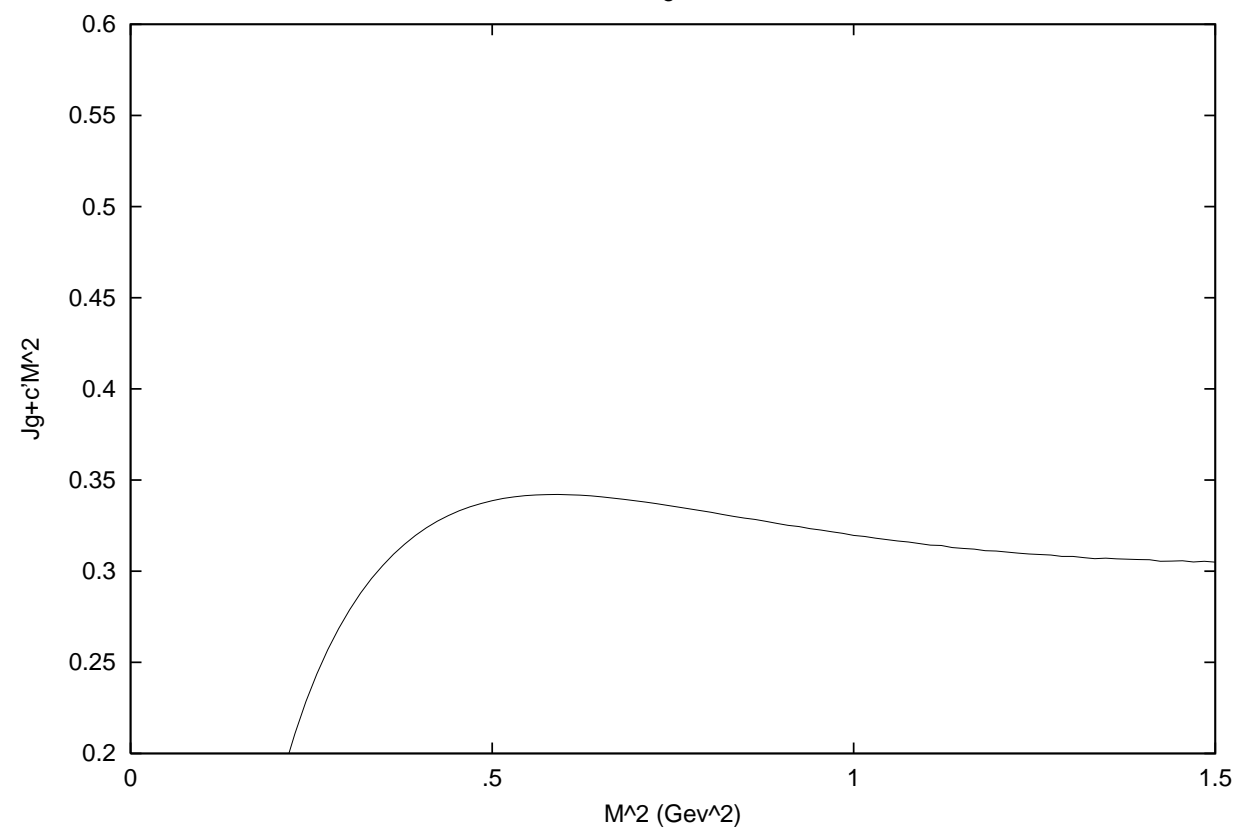




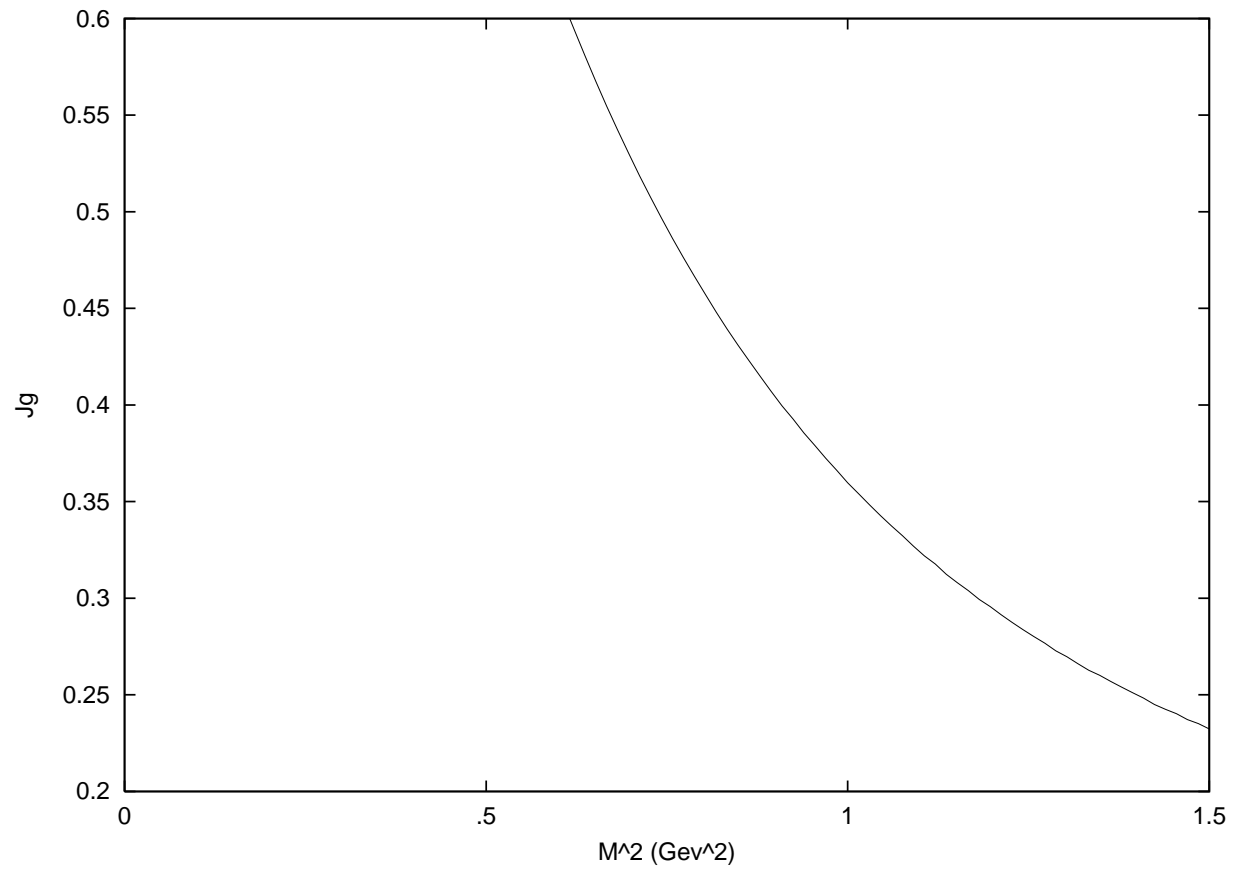




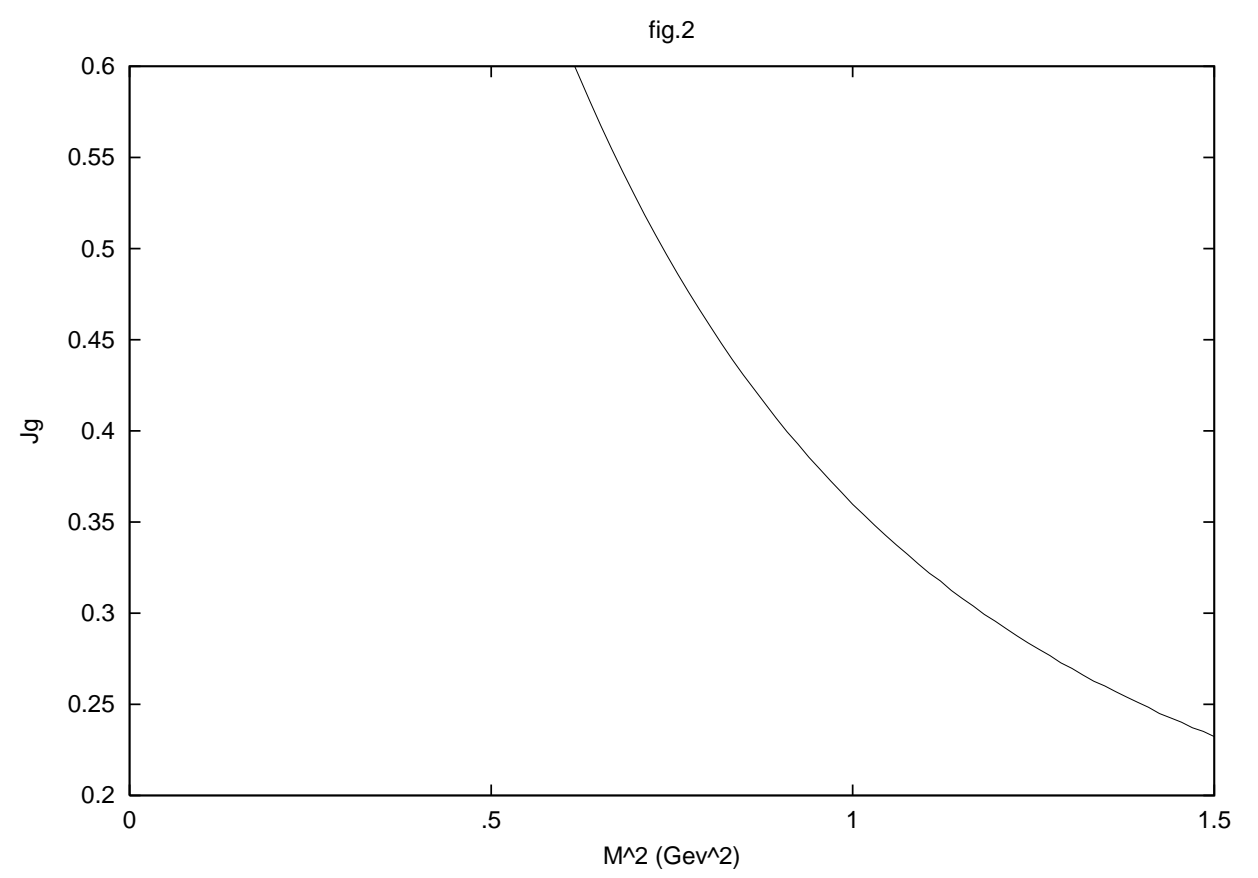

